\title{
STUDIES ON THE PERMEABILITY OF THE BLOOD-RETINAL BARRIER \\ II. BREAKDOWN OF THE BLOOD-RETINAL BARRIER BY INJURY*
}

\author{
BY \\ J. G. CUNHA-VAZ $\dagger$ \\ Department of Pathology, Institute of Ophthalmology, University of London
}

MajNo, Palade, and Schoefl (1961), revising an old technique, demonstrated that colloidal carbon injected intravenously was a good indicator of increased vascular permeability and clearly labelled the particular vessels through which plasma protein was escaping. These authors, using this technique of vascular labelling and studying the rat cremaster muscle, found that the increase in permeability after local application of histamine and serotonin was located electively in the venules and small veins. From their studies they concluded that all tissues of the body would probably react in a similar way.

Recently, Ashton and Cunha-Vaz (1965) applied this technique in studying the response of ocular vessels to histamine and found a similar location of the permeability changes in the conjunctiva, iris, and large vessel layer of the choroid. In contrast, the retinal vessels were found to be unique among the ocular vessels in being totally unaffected by histamine, and this was found to apply also to the cerebral vessels.

Of the many tissue changes which occur in response to injury, those of the blood vessels are fundamental, and include an increase in endothelial permeability permitting the passage of large molecules. Sevitt (1964) studied this permeability response and divided it into three phases: immediate, early, and delayed. The immediate phase being of short duration was mediated by histamine and other known endogenous mediators (Wilhelm and Mason, 1960; Majno, 1964); the early phase was presumably due to direct injury to the endothelium; the delayed phase was considered to be due to a mediator, but not to histamine or histamine-like substances (Wilhelm, 1962; Spector and Willoughby, 1963). Majno (1964) and Cotran and Majno (1964) showed the carbon labelling in the immediate phase to be venular, and in the delayed phase to be electively in the capillaries.

Our observation that histamine does not increase the permeability of the retinal vessels and the fact that histamine is accepted as playing an important role in the vascular response to injury in most tissues of the body seem to warrant a detailed study of the response to injury of the retinal vessels.

The rat was the animal chosen because of the characteristic disposition of its retinal vessels which is most suitable for a topographical study of the vascular tree. As shown by Ashton and Blach (1961), the retinal vessels of the rat are distributed in two layers, one superficial and another deep connected by branches of the superficial net which dip down sharply into the retina to join the deep capillary net. The

* Received for publication October 5, 1965.

$\uparrow$ Fellow of the Instituto de Alta Cultura, Portugal. On leave from the Department of Ophthalmology, University of Coimbra, Portugal. 
superficial net, located in the nerve fibre layer, is almost entirely arterial in character, with no intercommunications with the veins in this layer. The deeper net, in contrast, is entirely venous and drains into tributaries, the largest being peripheral, which travel steeply towards the surface of the retina to the main veins.

\section{Material}

Male albino rats were used in these experiments. They were anaesthetized with subcutaneous sodium barbitone after a previous intraperitoneal injection of Largactil. When necessary ether was used to deepen the anaesthesia. In some experiments ether alone was used.

\section{Injurious Agents}

Trauma.-A 30-gauge needle was inserted into the vitreous and the retina touched with the point under microscopical observation.

Thermal Injury.-The fine point of an electrocautery was applied to the sclera and contact maintained until a small drop of vitreous escaped.

Chloroform + Methanol.-A mixture of chloroform and methanol was prepared in a proportion of two parts of the first to one part of the second as indicated by Purpura, Girado, Smith, and Gomez (1958).

Glycerol.-This was used in its pure form.

Colloidal Carbon.-An identical preparation to that described in previous papers (Ashton and Cunha-Vaz, 1965; Cunha-Vaz, Shakib, and Ashton, 1966) was used. This contains $100 \mathrm{mg} . / \mathrm{ml}$. carbon with average particle size of $200 \AA$. The standard dose for intravenous injections was $0 \cdot 1 \mathrm{ml} . / 100 \mathrm{~g}$. body weight.

\section{Methods}

\section{Local Application of Injurious Agents to the Retina}

In most experiments the injurious agents were injected into the vitreous under microscopical observation. The eye was immobilized with two stitches in the sclera near the limbus and a small coverslip was applied on the cornea and the space between the cornea and coverslip filled with saline. The size of the coverslip was made to be small enough to leave room for a 30-gauge needle to be introduced into the vitreous through the sclera without changing the position of the coverslip and thus allowing continuous observation of the retina at a $10 \times$ magnification with a Zeiss-Opton microscope. A drop of the injurious agent was then placed in direct contact with the retina.

In other experiments, to ensure a direct contact of the agents with the retina, they were applied under microscopical observation after removing the cornea, lens and vitreous.

In the first experiments, the entire vascular system of the animal was irrigated via the left ventricle with heparinized saline to remove any carbon that might have remained in the lumen of the vessels. Later, this procedure was discontinued because of the fact that one hour after the colloidal carbon injection, when the animals were killed, no carbon particles remained in the retinal circulation of the control eyes; this was expected from the studies of Halpern, Benacerraf, and Biozzi (1953), which showed that at this time all the carbon has been taken up by the reticulo-endothelial system.

Colloidal Carbon Injections. - To demonstrate the leaking vessels, the colloidal carbon suspension was injected into the femoral vein immediately before the local application of the injurious agents or after at intervals of 2, 4, 12, and 24 hours in different animals. 


\section{Preparation of the Specimens for Microscopical Examinations}

The eyes were excised and placed in 10 per cent. formol saline. The retinae were removed after 24 hrs' fixation, mounted flat, and cleared in glycerin. After examination and photograph, some of the retinae were digested by the method of Kuwabara and Cogan (1960) as modified by Ashton (1963) and were subsequently stained by periodic-acid Schiff haematoxylin (PASH); the others were embedded in paraffin wax, and transverse and flat sections were stained with haematoxylin and eosin or PASH.

\section{Controls}

The material described in a previous paper (Ashton and Cunha-Vaz, 1965) served as control for the present studies.

\section{Results}

\section{Vascular Labelling after Local Application of Chloroform +Methanol on the Retina}

When carbon was injected immediately before the local application of chloroform + methanol to the retina, and the animals were killed 1 hour after the injection, the retinae showed an extensive blackening of almost all retinal vessels in the area of contact of the chloroform + methanol with the retina (Fig. 1). This vascular labelling was found in all vessels regardless of type and calibre. The carbon deposits were always sharply confined to the walls of the affected vessels and the distribution of the carbon particles in the vessel walls was irregular and impossible to relate to any fixed pattern. Sometimes the deposits were so dense that the vessels appeared as black tubules.

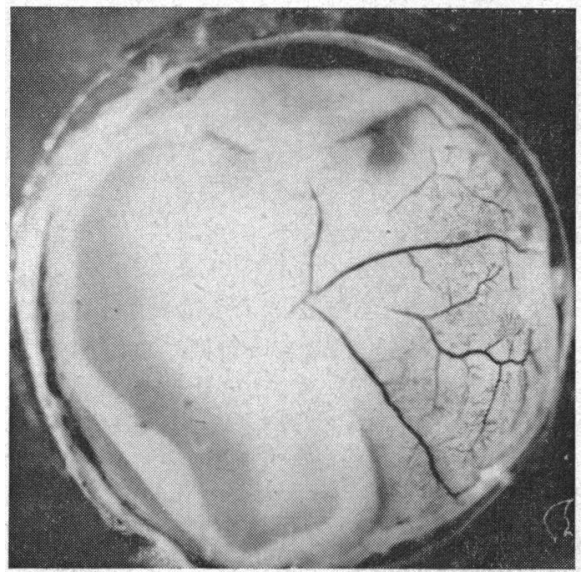

FIG. 1.-Opened eye of rat, showing extensive blackening of all retinal vessels, regardless of calibre, in the area of contact of chloroform + methanol with retina. The carbon was injected immediately before the local application of chloroform + methanol. $\times 10$.

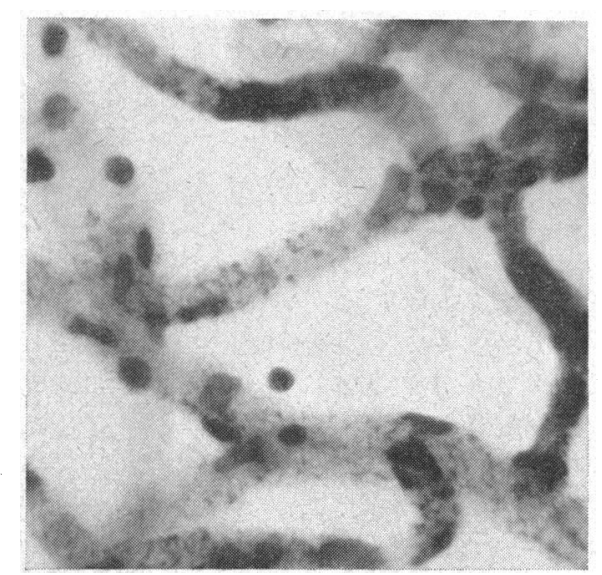

FIG. 2.-Digested retina from a rat in which carbon was injected immediately before the local application of chloroform + methanol. Note widespread deposition of carbon deposits in vessel walls showing absence of any definite pattern. PASH. $\times 620$.

In the digested retinae, a generalized and widespread vascular labelling could be observed in the area of injury (Fig. 2). The endothelial cells, which could be observed only when they were not masked completely by the carbon deposits, s̀nowed a pale staining. The vessel walls showed increased staining with PAS. Histologically, the retinae showed oedema, but no significant exudation of leucocytes. 
When carbon was injected 2 and 4 hrs after the local application of chloroform + methanol, and the animals were killed after 1 hour had elapsed, the retinae presented a characteristic distribution of vascular labelling (Fig. 3). The vessels in the zone of direct contact of the chloroform + methanol with the retina now showed a complete absence of carbon labelling. Most of the vessels in this area were engorged and packed with red cells and presented numerous dilatations, undoubtedly as a result of severe stasis (Fig. 4).

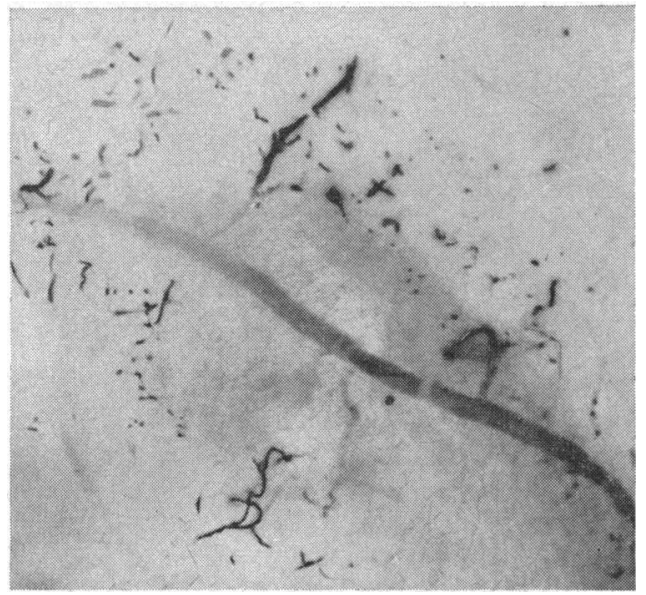

FIg. 3.-Cleared retina $2 \mathrm{hrs}$ after local application of chloroform + methanol. There is a central zone where no vascular labelling can be seen; the vessels in this area are full of red cells. Around this central zone is a relatively narrow border where almost all vessels, regardless of calibre, are labelled with carbon. $\times 32$.

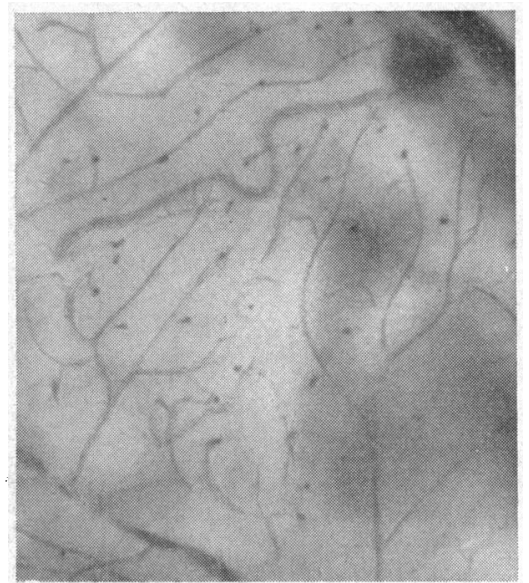

Fig. 4.-Cleared retina $4 \mathrm{hrs}$ after local application of chloroform + methanol, showing centre of lesion with vessels presenting a severe degree of stasis. $\times 56$.

Vascular labelling was now confined to a very narrow rim of vessels of all calibres around the lesion (Fig. 5, overleaf).

Digested retinae showed that the vessels in the central area, where no vascular labelling could be seen, presented a widespread loss of endothelial cells and intramural pericytes and their walls were intensely PAS-positive. Those in the peripheral narrow rim of labelling (Fig. 6, overleaf), had endothelial cells with pale-staining nuclei and there were figures of migration in the intramural pericytes (Oliveira, 1966). Histologically, the central area presented intense oedema and signs of impending necrosis of the retinal cells. In the narrow peripheral zone, the retinae retained most of their normal structure but the oedema was intense and migration of leucocytes could be observed. Most of the vessels in the central area showed thickened walls intensely stained with PAS, cell loss, and signs of thrombosis. The labelled vessels in the peripheral area were patent.

In both groups of animals injected with carbon 12 and 24 hrs after the local application of chloroform +methanol, a central area of static vessels and a narrow rim of labelled vessels were seen as in the 2 and 4-hr specimens. In the peripheral area of vascular labelling, however, the carbon deposits could now be seen spreading out from the vessel walls into the adjacent tissue, an appearance referred to before as "smudging". This peripheral area showed at this stage a slight predominance of vascular labelling in the vessels of the deep net, that is in the venous side of the circulation, most probably due to the advanced degree of stasis of the neighbouring vessels. 


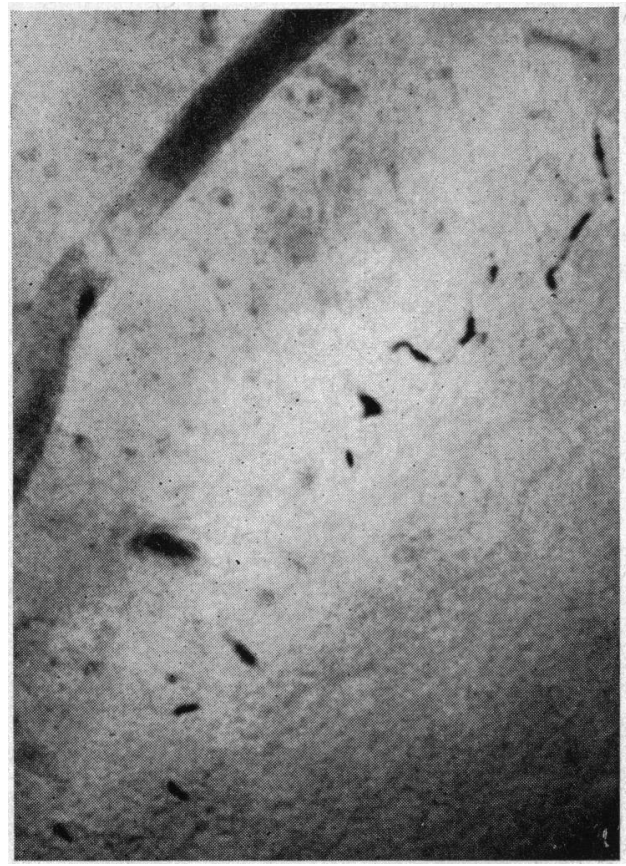

FIG. 5.-Cleared retina 4 hrs after local application of chloroform + methanol. Zone of labelling limited to a very narrow rim surrounding centre of lesion (on the left). $\times 110$.

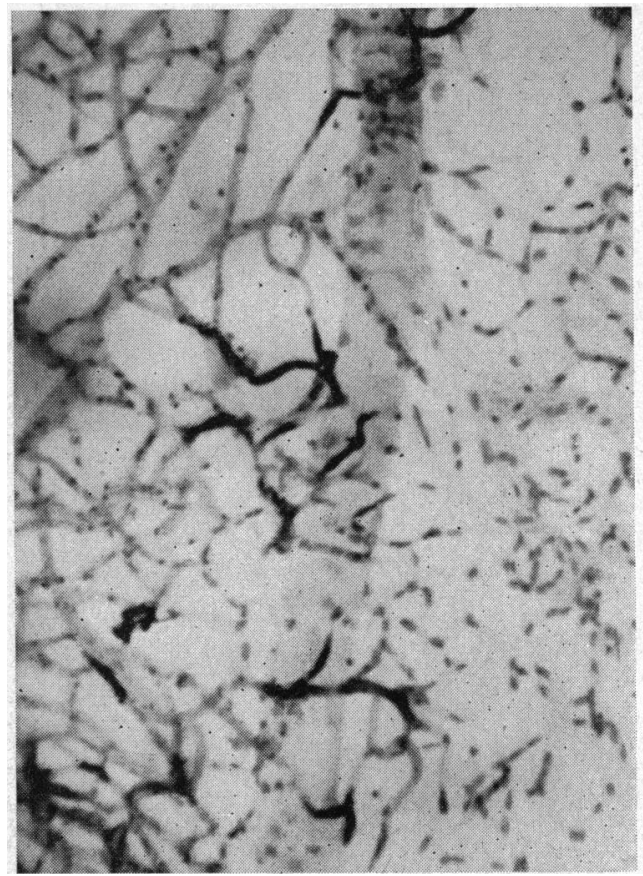

FIG. 6.-Digested retina 4 hrs after local application of chloroform + methanol. In the centre are labelled vessels belonging to peripheral rim of vascular labelling. On the left is part of central area of lesion with vessels showing intense deposition of PAS-positive material in the walls. On the right the retinal vessels are normal. $\times 110$.

Digested retinae showed an almost complete cell loss from the vessels of the central area. Histologically, the more damaged area of the retinae showed necrosis and occlusion of most of the vessels. There were numerous macrophages in the retinal

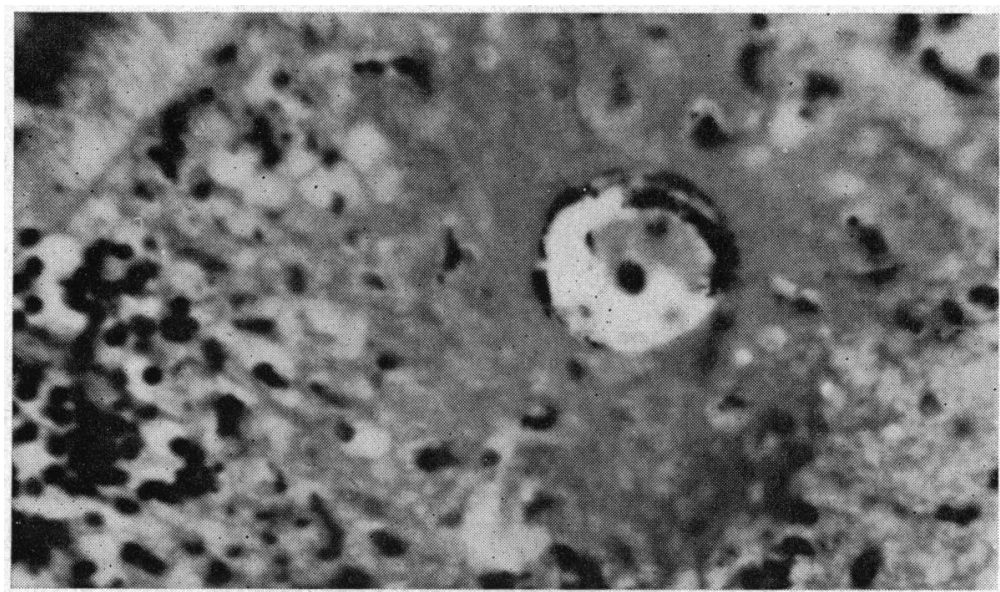

Fig. 7.-Section of retina, $24 \mathrm{hrs}$ after local application of chloroform + methanol, through peripheral rim of vascular labelling. The vessel lies in the inner nuclear layer and shows carbon deposition in the wall with extension into the adjacent tissues. Note intense exudation of PAS-positive material from the vessel. $\times 700$. 
tissue. PAS-positive material could be seen leaking from the labelled vessels of the peripheral narrow rim (Fig. 7, see p. 458).

\section{Vascular Labelling after Local Application of Glycerol on the Retina}

The sequence of events observed with chloroform + methanol could also be seen in the experiments with glycerol. The lesion produced by the application of glycerol, however, was generally less intense and the central area of severe damage was sometimes missing. In milder lesions, only the superficial vascular net showed evidence of carbon leakage, but even so the vessels were labelled regardless of their calibres (Fig. 8).

(8)
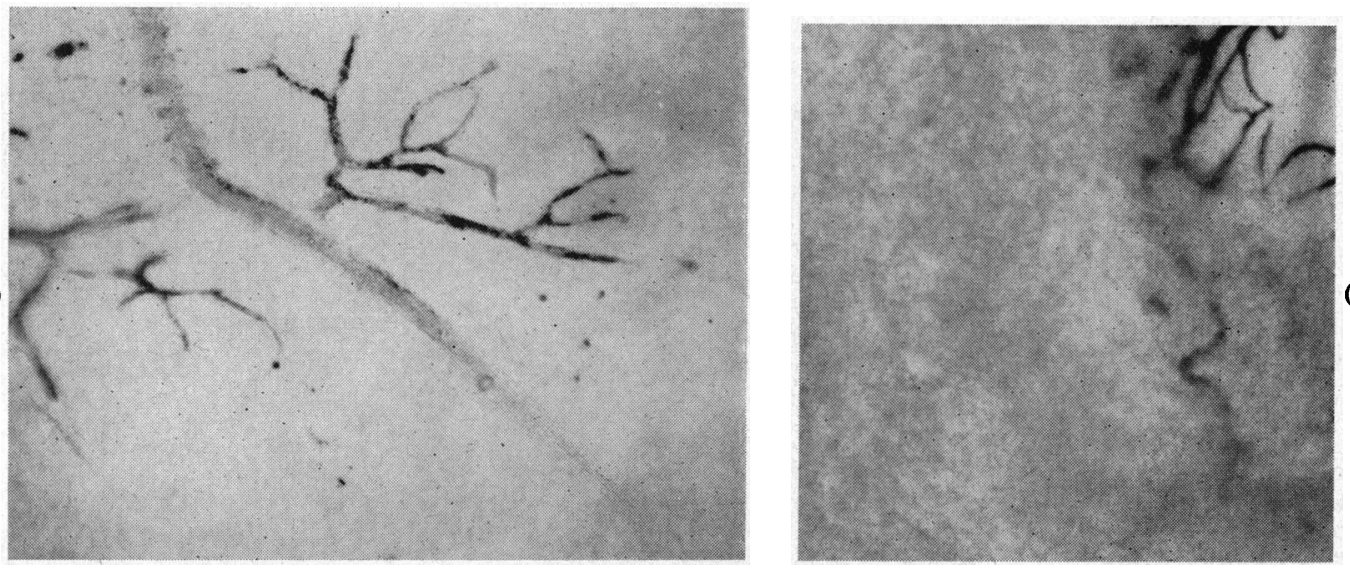

(9)

Fig. 8.-Cleared retina, showing mild lesion produced by local application of glycerol. The colloidal carbon was injected immediately before the application of glycerol. The vascular labelling is limited to the superficial net in the large and small arterioles. $\times 110$.

Fig. 9.-Cleared retina injected with carbon immediately before the local application of heat. The vascular labelling is limited to the vessels directly damaged by the thermal injury (right). $\times 85$.

\section{Vascular Labelling after Thermal Injury to the Retina}

Here only the vessels directly damaged by the heat application presented vascular labelling, again regardless of calibre (Fig. 9).

This vascular labelling was evident when the colloidal carbon was injected before or immediately after the thermal lesion was produced. When carbon was injected later there was almost no labelling because of stasis of the vessels directly injured.

\section{Vascular Labelling after Traumatization of the Retina}

The results obtained in this group were similar to those already described under the heading of thermal injury.

\section{Discussion}

The colloidal carbon technique used in the present investigation has revealed that the increased vascular permeability which results from chemical, thermal, and traumatic injury of the retina follows a consistent pattern, characterized by involvement of all vessels, regardless of type and calibre, in direct contact with the injurious agent. 
The leakage from the affected vessels is immediate and leads to severe, often irreversible, stasis. Around this central zone of immediate labelling, a delayed phase of vascular labelling could be observed in a small peripheral area, but in this phase also the vessels were affected regardless of type or calibre. No phase of specific increased venular labelling could be observed, and as all substances at present known to increase vascular permeability induce carbon leakage only in venules (Majno and others, 1961; Hurley and Spector, 1961 ; Majno, 1964), the present findings are against the possibility that any known endogenous mediators play a role in the retinal vascular response to injury. The evidence favours the view that increased vascular permeability in the retina as a result of injury can be brought about only by direct damage to the endothelial cell itself.

It was recently reported by Ashton and Cunha-Vaz (1965) that, in contrast with the other ocular vessels which showed vascular labelling after local application of histamine, the retinal vessels were unique in being totally unaffected by histamine. The absence of venular labelling in the retinal vessels after local application of histamine or after various forms of injury, as reported here, indicates either that histamine is not released by injury to the retina or that it is not effective because of peculiar characteristics of the retinal vessels.

Various hypotheses have been proposed to explain the elective increase in venular permeability to histamine and other mediators in most tissues of the body. Majno (1964) and Movat and Fernando (1964) believe the small venules to have special properties of their own, under normal circumstances as well as under pathological conditions, and these authors have looked for an ultrastructural difference without much success. That there are probably no permanent structural differences in the vessel wall was shown by Zweifach (1940), who found by re-routing the circulation in an opposite direction that the increased permeability area was reversed towards the arterial side of the circulation. Another interesting explanation was given by Rowley (1963), who showed that histamine, serotonin, bradykinin, and 48/80 produced a segmental constriction of veins and distension of venules draining into the constricted segments. He suggested that the increased permeability in the venules was a consequence of raised intraluminal pressure. This author demonstrated intercellular carbon leakage in the venules of the paw after elevation of venous pressure in perfused hind limbs of rats.

In the retina, the extensive electron microscopical studies of retinal vessels of different experimental animals in progress in this laboratory have failed to show any difference between venules and capillaries, but they have presented us with evidence of a difference between the junctional attachments of the retinal vessels and other vessels of the body, which could explain the impermeability of the retinal vessels to histamine (Cunha-Vaz and others, 1966). Regarding the explanation proposed by Rowley (1963), it is interesting that the retinal veins do not possess smooth muscle cells, but only widely-spaced intramural pericytes which most probably cannot contract (Wiedeman, 1963).

The possibility that histamine is not released in the retinal response to injury cannot be ruled out, as there is evidence that histamine is liberated in most tissues of the body by a large variety of chemical substances (Miles and Wilhelm, 1960) and by thermal injury (Majno, 1964). It is interesting that the delayed phase of elective capillary 
labelling reported after thermal injury by Wells and Miles (1963), Sevitt (1964), Majno (1964), and Cotran and Majno (1964), and attributed by these authors to liberation of an unknown mediator, was similarly not found in the retina.

Another interesting finding was the absence in the labelled retinal vessels of the characteristic mosaic pattern observed by Majno and others (1961) in the cremaster and by Ashton and Cunha-Vaz (1965) in the vessels of the iris, which as shown in the electron microscopical studies by Majno and Palade (1961) is due to preferential leakage through the intercellular junctions. In the present study, the leaking vessels showed a most irregular distribution of the carbon particles in their walls with no elective relation to the intercellular junctions. This observation is in agreement with the recent work of Cunha-Vaz and others (1966), who showed that these junctions are exceptionally adherent.

Finally, this study shows a similar behaviour of the Blood-Retinal Barrier and Blood-Brain Barrier to different forms of injury, and emphasizes the importance of their vascular component. Bakay (1955), studying stab wounds in the brain with the radioisotope P32, observed that the peak of local barrier breakdown was reached immediately after the injury and was most probably related to direct capillary damage, which could be demonstrated in our experiments. Also, more recently, Clasen, Cooke, Pandolf, Boyd, and Raimondi (1962) and Bakay and Haque (1964), studying the effects of cold injury in the brain, compared these experimental lesions to the conditions seen in human disease (as in haemorrhagic infarcts, cerebral contusion, and brain tumours) and stressed that exudation of protein through hyperpermeable blood vessels is an unequivocal part of the process of focal cerebral oedema in injury.

\section{Summary}

The pattern of increased vascular permeability in the retina after chemical, thermal, and traumatic injury was followed after the injection of colloidal carbon in vivo; this technique leads to the labelling of vessels in a state of increased permeability to circulating particles, the carbon accumulating between endothelium and basement membrane or intramural pericyte.

The evidence obtained shows that increased vascular permeability in the retina as a result of different forms of injury can be brought about only by direct damage to the endothelium itself. No phase of specific increased venular labelling could be observed, a fact which eliminates the possibility of any known endogenous mediators playing a role in the retinal vascular response to injury.

Finally, the response of the Blood-Retinal Barrier to different forms of injury is compared with that of the Blood-Brain Barrier, and the importance of their vascular component is emphasized.

I am deeply indebted to Prof. Norman Ashton for his suggestions and interest in this work. I should also like to thank Miss E. Robins and Miss N. Yarkoff for their technical assistance. 


\section{REFERENCES}

Ashton, N. (1963). Brit. J. Ophthal., 47, 521. and BLACH, R. (1961). Ibid., 45, 321.

and Cunha-VaZ, J. G. (1965). Arch. Ophthal. (Chicago), 73, 211.

BAKaY, L. (1955). (A.M.A.) Arch. Neurol. Psychiat., 73, 2. and HAQUE, I. U. (1964). J. Neuropath. exp. Neurol., 23, 393.

Clasen, R. A., CoOke, P. M., Pandolfi, S., Boyd, D., and Raimondi, A. J. (1962). I Ibid., $21,579$.

Cotran, R. S., and Majno, G. (1964). Amer. J. Path., 45, 261.

Cunha-Vaz, J. G., Shakib, M., and Ashton, N. (1966). Brit. J. Ophthal., 50, 441.

Halpern, B. N., Benacerraf, B., and Biozzi, G. (1953). Brit. J. exp. Path., 34, 426.

HURLeY, J. V., and SPECTOR, W. G. (1961). J. Path. Bact., 82, 421.

Kuwabara, T., and Cogan, D. G. (1960). Arch. Ophthal. (Chicago), 64, 904.

MAJNO, G. (1964). In "International Symposium on Injury, Inflammation, and Immunity", ed. L. Thomas, J. W. Uhr, and L. Grant, pp. 58-93. Williams and Wilkins, Baltimore.

- and Palade, G. E. (1961). J. Biophys. biochem. Cytol., 11, 571.

,$- \longrightarrow$, and SCHOEFL, G. I. (1961). Ibid., 11, 607.

*Miles, A. A., and WilHeLm, D. K. (1960). In "The Biochemical Response to Injury", ed. H. B. Stoner, pp. 51-83. Blackwell, Oxford.

Movat, H. Z., and Fernando, N. V. P. (1964). Exp. molec. Path., 3, 98.

Oliveira, F. (1966). Brit. J. Ophthal., 50, 134.

Purpura, D. P., Girado, M., Smith, T. G., and Gomez, J. A. (1958). Proc. Soc. exp. Biol. (N. Y.), 97,348

RowLey, D. A. (1963). Brit. J. exp. Path., 44, 284.

SEVITT, S. (1964). In "International Symposium on Injury, Inflammation, and Immunity", ed. L. Thomas, J. W. Ure, and L. Grant, pp. 183-210. Williams and Wilkins, Baltimore.

Spector, W. G., and WilloughBY, D. A. (1963). Bact. Rev., 27, 117.

WelLS, F. R., and Miles, A. A. (1963). Nature (Lond.), 200, 1015.

Wiedeman, M. P. (1963). In "Handbook of Physiology, Sect. II-Circulation", ed. W. F. Ha milton and P. Dow. American Physiological Society, Washington.

Wilhelm, D. L. (1962). Pharmacol. Rev., 14, 251. - and Mason, B. (1960). Brit. J. exp. Path., 41, 487.

ZWEIFACH, B. W. (1940). Cold Spr. Harb. Symp. quant. Biol., 8, 216.

* A Symposium organized by the Council for International Organizations of Medical Sciences. 